

\title{
Anomalous scaling in random shell models for passive scalars
}

\author{
A. Wirth $^{1} \&$ L. Biferale ${ }^{2}$ \\ November 18, 2018 \\ ${ }^{1}$ CNRS, Observatoire de Nice, B.P. 229, 06304 Nice Cedex 4, France. \\ ${ }^{2}$ Universitá di Roma "Tor Vergata", Dip. di Fisica \\ Via della Ricerca Scientifica 1, 00133 Roma, Italy.
}

\begin{abstract}
A shell-model version of Kraichnan's (1994 Phys. Rev. Lett. 72, 1016) passive scalar problem is introduced which is inspired from the model of Jensen, Paladin and Vulpiani (1992 Phys. Rev. A45, 7214). As in the original problem, the prescribed random velocity field is Gaussian, delta-correlated in time and has a power-law spectrum $\propto k_{m}^{-\xi}$, where $k_{m}$ is the wavenumber. Deterministic differential equations for second and fourth-order moments are obtained and then solved numerically. The second-order structure function of the passive scalar has normal scaling, while the fourth-order structure function has anomalous scaling. For $\xi=2 / 3$ the anomalous scaling exponents $\zeta_{p}$ are determined for structure functions up to $p=16$ by Monte Carlo simulations of the random shell model, using a stochastic differential equation scheme, validated by comparison with the results obtained for the second and fourthorder structure functions.
\end{abstract}

ROM2F $/ 96 / 29$

\section{Introduction}

A most striking property of a passive scalar quantity advected by a fully developed turbulent flow

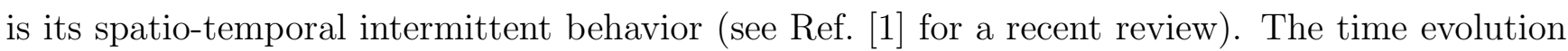
of the scalar field $\theta$ is described by the partial differential equation:

$$
\partial_{t} \theta+(\mathbf{v} \cdot \nabla) \theta=\kappa \Delta \theta+f,
$$

where $\mathbf{v}$ is the advecting velocity field, $\kappa$ is the molecular diffusivity and $f$ is an external forcing. 
Intermittency in passive scalars advected by highly chaotic flows is thought to be connected to the existence of self-similar processes transferring fluctuations from large to small scales.

Self-similarity is observed in the power-law behavior of structure functions, $\tilde{S}_{p}(r)$, in the inertial range, that is moments of $\theta$-increments at scales where neither external forcing nor molecular damping are acting:

$$
\tilde{S}_{p}(r) \equiv\left\langle|\theta(x)-\theta(x+r)|^{p}\right\rangle \sim r^{\zeta_{p}} .
$$

The set of scaling exponents, $\zeta_{p}$, fully characterizes intermittency. In particular, deviations from the dimensional (linear) behavior $\zeta_{2 p}=p \zeta_{2}$ are evidence of a nontrivial scalar transfer among scales, in analogy to the energy intermittent cascade in turbulent flows (see Ref. [2] for a recent review on this subject).

Another important issue is connected to the questioned universal character of scalar fluctuations in the inertial range. Universality should be the consequence of local, or quasi-local, interactions among scales and it should be reflected in a strong robustness of the scaling exponents, $\zeta_{p}$, against variation of forcing and/or dissipation mechanisms. This idea dates back to the phenomenological work of Obukhov and Corrsin [3].

Statistics of the passive scalar must be strongly related to the properties of the advecting velocity fields; therefore, the only realistic case would be to study the "full problem" given by (1) together with the corresponding Navier-Stokes equations, in the limit of high Reynolds number, which describes the time evolution of a fully developed turbulent velocity field.

Due to the lack of knowledge about the statistical properties of a fully developed turbulent flow the "full problem" cannot yet be attacked. Nevertheless, equation (1) with a prescribed velocity field of suitable statistical properties, could be a good "training ground" for understanding general mechanisms (if any) underlying the presence of anomalous scaling in fluid-dynamics.

Recently, much attention has been paid in this direction by studying the intermittency properties of a passive scalar advected by a velocity field which is Gaussian, white-in-time and which has self-similar spatial correlations. In Refs. \&4 6, 7, 8, 9, 12, 10 the two-point velocity field correlation function was set to: $\left\langle v_{i}(x, t) v_{j}\left(x^{\prime}, t^{\prime}\right)\right\rangle=\delta\left(t-t^{\prime}\right) D_{i j}\left(x-x^{\prime}\right)$, with $D(x)=D(0)-\hat{D}(x)$. Here, $\hat{D}$ is the $d$-dimensional velocity-field structure function:

$$
\hat{D}_{i j}(x)=D_{0}|x|^{\xi}\left[(d-1+\xi) \delta_{i j}-\xi x_{i} x_{j}|x|^{-2}\right]
$$

where the scaling exponent $\xi$ of the second order velocity structure function, $\xi$, with $0<\xi<2$, is a free parameter. Higher order velocity-field correlation functions are fixed by the Gaussian assumption.

Such a choice is far from being realistic because of the assumed fast-time-decorelation and Gaussianity. Therefore, no quantitative agreement with the intermittent properties of the "full problem" must be expected. Nevertheless, in Refs. [⿴囗, 6, 7, 8, 10, 11] many interesting analytical and phenomenological results for this toy-model have been obtained, giving for the first time the impression that some new insight into the "intermittency problem" has been gained (see Ref. [13] for related results in a passive vector case).

The big advantage of this toy-model is that, due to the delta-correlation in time, all equations for equal-time $n$-points correlations are formally closed, that is, the $n$-th-order correlation depends 
only on lower order correlations. This fact was stressed by Kraichnan [ [4, 5], who gave for the first time the closed expression for $\tilde{S}_{2}(r)$. In Ref. [4 a theory for all structure functions was proposed and an explicit formula for $\zeta_{p}$ given. The main physical outcome was that all structure functions of order greater than two have intermittency corrections and that intermittency should be connected to some non trivial matching between advective and diffusive properties of the model. Indeed, for structure functions of order greater than two it is no more possible to clearly separate the contributions from diffusion and advection terms in the generation of anomalous scaling.

In Refs. [6, 8, 10] it has been shown that intermittency of the scalar structure functions is connected to the properties of the null-space of the linear operators describing the inertial-scale evolution of multipoint passive scalar moments. Moreover, perturbative expressions of intermittency corrections, as a function of the parameter $\xi$ and as a function of the inverse of the dimensionality of the system were given [6, 8, 10]. In both cases careful analysis of matching conditions at infrared (IR) and ultraviolet (UV) scales must be taken into account. Universality in the scaling exponents is still preserved, while constants in front of the power laws acquire dependencies on the forcing and on the passive scalar dissipation. How far all these results can be freely extended to the "full problem" is difficult to say. Certainly, the scaling exponents have a strong quantitative dependence on the statistical properties of the prescribed velocity field. Influence of a short-but-non-zero correlation time was studied in Ref. [9] and found important, while from a phenomenological point of view it is difficult to say if the perturbation expansion of Ref. [6] can be extended up to the value $\xi=2 / 3$ which mimics the spatial energy spectrum of true turbulence. (Let us notice that, even for $\xi=2 / 3$, the assumed delta-correlation in time for the velocity makes the classical Obukhov-Corrsin theory inapplicable to the passive scalar two-point correlation function [5].)

An important drawback of the Gaussian delta-correlated toy-model is that numerical simulations seem even more difficult than a direct numerical simulation of the "full problem". This is because (1) is a stochastic differential equations (SDE) the solution of which requires huge computer resources, because of the massive use of random number generators and also because of the paramount difficulties in the implementation of higher than first order discretization schemes [22]. The state of the art of SDE numerical simulations is hardly compatible with the high spatial resolution needed to study scaling properties of the solution of (四) with a white-in-time velocity field (see however Ref. [7] for a first attempt at overcoming these difficulties, albeit at the prize of introducing some anisotropy into the velocity field).

In this spirit, we have tried to work with the simplest toy-model which has some connection to the physics of the "full problem", while at the same time aiming for something more tractable analytically and/or numerically. We have thus investigated the intermittency properties of a shellmodel for a passive scalar advected by a prescribed stochastic velocity field. Shell models (see Ref. [14] for a pedagogical introduction) have been already successful in helping to understand many issues connected to fully developed turbulence [15, 16, 17, 18, 19, 20].

The problem of defining a shell model for the advection of a passive scalar by a deterministic and chaotic velocity field has been already investigated in Ref. [21]. Here, we are going to present our numerical and analytical results obtained in a weakly modified version of the shell model defined in Ref. [21], but using a delta-correlated surrogate for the advecting velocity field. The 
main drawback of our model is that, following shell-model philosophy, it retains only nearest and next-nearest neighbor interactions in the shell index. Having only semi-local interaction in the Fourier space could be a problem for describing the physics of passive fluctuations for those types of velocity-field-correlations which induce strong non-local effects. For example, simple dimensional considerations tell us that the limit of very small $\xi$ should be dominated by strong non-local effects (which leads to an ultraviolet divergence of the eddy-diffusivity in the limit $\xi \rightarrow 0$ [6]). Therefore we do not expect that our shell model could properly mimic the physics of the PDE (11) in the range of parameters choice $0<\xi \ll 1$. Yet, we are quite confident that our model captures very well the passive scalar physics for $\xi=O(1)$, that is when dominant interactions become more local in Fourier space, as is the case for true turbulence. The major advantage of using a shell-model is that, now, reliable numerical simulations become feasible, allowing nonperturbative determinations of scaling exponents up to high orders (in this paper we present results up to order 16). Furthermore, having reliable numerical simulations should open the possibility of relaxing some of the constraints put on the statistical properties of the velocity field, thereby allowing investigation of increasingly realistic problems.

We found that our shell model shares many properties with the original SDE (1). Among them, the most importants are: (i) the second order structure function has normal scaling, (ii) all structure functions of order larger than two have anomalous corrections, (iii) there is a remarkable coupling between both UV and IR scales with inertial terms in the closed equation satisfied by fourth-order shell correlations. This result suggests that the anomalous scaling is due to nontrivial contributions of integral and diffusive scales on the inertial-range properties. Moreover, we find that anomalous behavior tends to vanish when approaching the laminar regime, $\xi=2$, for the advecting velocity field.

Even though we have not obtained an analytical expression for the anomalous scaling exponents, we are confident that many useful insights can be obtained by further investigation of the present model, or even simpler one. For example, it should be possible to define some exactly solvable models, where the shell-velocity correlations is chosen such as to give exactly solvable (but nontrivial) linear operators describing the structure functions inertial properties

The paper is organized as follows: in Section 2 we introduce the random passive scalar shell model and we discuss some problems connected with its stochastic differential equation (SDE) formulation. In Section 3 we explicitly derive the closed deterministic equations for second and

fourth-order moments, which are numerically solved in Section 4 to obtain the scaling exponents of second and fourth-order structure functions. Monte Carlo simulations of the SDE are presented in Section 5. Concluding remarks and suggestions for further work are given in Section 6 .

\section{The model and its stochastic differential equation for- mulation}

We recall the main features of the passive scalar shell model of Ref. [21]. The model is defined in terms of a shell-discretization of the Fourier space in a set of wavenumber defined on a geometric 
progression $k_{n}=k_{0} 2^{n}$. Passive increments at scale $r_{n}=k_{n}^{-1}$ are described by a complex variable $\theta_{n}(t)$. The time evolution is obtained according to the following criteria: (i) the linear term is a purely diffusive term given by $-\kappa k_{n}^{2} \theta_{n}$; (ii) the advection term is a combination of the form $k_{n} \theta_{n^{\prime}} u_{n^{\prime \prime}}$; (iii) interacting shells are restricted to nearest and next-nearest neighbors of $n$; (iv) in the absence of forcing and damping the model conserves the volume in the phase-space and the passive-energy $E=\sum_{n}\left|\theta_{n}\right|^{2}$. Properties (i), (ii) and and (iv) are valid also for the original equation (1) in the Fourier space, while property (iii) is an assumption of locality of interactions among modes, which is rather well founded as long as the power law spectra of the passive scalar $E(k) \sim k^{-\alpha}$ has $1<\alpha<3$.

Our passive scalar shell model, inspired by Jensen, Paladin and Vulpiani's model [21], is defined by the following equations $(m=1,2, \ldots)$

$$
\begin{aligned}
{\left[\frac{d}{d t}+\kappa k_{m}^{2}\right] \theta_{m}(t) } & =i\left[a_{m}\left(\theta_{m+1}^{*}(t) u_{m-1}^{*}(t)-\theta_{m-1}^{*}(t) u_{m+1}^{*}(t)\right)\right. \\
& +b_{m}\left(\theta_{m-1}^{*}(t) u_{m-2}^{*}(t)+\theta_{m-2}^{*}(t) u_{m-1}(t)\right) \\
& \left.+c_{m}\left(\theta_{m+2}^{*}(t) u_{m+1}(t)+\theta_{m+1}^{*}(t) u_{m+2}^{*}(t)\right)\right]+\delta_{m 1} f(t)
\end{aligned}
$$

where the star denotes complex conjugation and

$$
k_{m}=2^{m}, \quad a_{m}=\frac{k_{m}}{2}, \quad b_{m}=-\frac{k_{m-1}}{2}, \quad c_{m}=\frac{k_{m+1}}{2},
$$

with $u_{-1}=u_{0}=\theta_{-1}=\theta_{0}=0$ as boundary conditions. The forcing term $\delta_{1 m} f(t)$ acts only on the first shell. Note that (5) is one of possible choices for the parameters $a_{m}, b_{m}$ and $c_{m}$ ensuring the conservation of passive scalar energy $\sum_{m} \theta_{m} \theta_{m}^{*}$ and phase-space volume. In numerical implementations, the model is truncated to a finite number of shells $N$ (here $N=19$ ) with the additional boundary conditions $u_{N+1}=u_{N+2}=\theta_{N+1}=\theta_{N+2}=0$.

Our model differs from that of Ref. [21] by the absence of complex conjugation on two of the $u$ factors in the rhs of (田). The reason for this change will become clear below. Furthermore, in Ref. [21] the passive scalar model (4) was coupled to the GOY shell model [15, 16, 17] for the nonlinear dynamics of the velocity variables. The GOY model displays multifractal behavior for the $u_{n}$ 's and an interesting intermittent behavior is also found for the passive scalar.

Our goal,as in Kraichnan's work [4], is to use a nonintermittent velocity field and then to find if the passive scalar is nevertheless intermittent. For this, we assume that the velocity variables $u_{m}(t)$ and the forcing term $f(t)$ are independent complex Gaussian and white-in-time, that is, delta-correlated. Furthermore, as in Ref. [5], we make a scaling assumption for the spectrum of the $u_{m}(t)$ 's, namely

$$
\left\langle u_{m}(t) u_{m}^{*}\left(t^{\prime}\right)\right\rangle=\delta\left(t-t^{\prime}\right) D_{m}
$$

where

$$
D_{m}=k_{m}^{-\xi}
$$

will be called the velocity spectrum. For $f(t)$ we assume $\left\langle f(t) f\left(t^{\prime}\right)\right\rangle=\delta\left(t-t^{\prime}\right)$. 
As long as the velocity variables $u_{m}(t)$ have a finite correlation time and, hence, smooth sample paths, there is no particular difficulty in giving a meaning to the set (4) of random ordinary differential equations (ODE's). A well-known difficulty arises with ODE's having white-in-time coefficients: the mathematical meaning of the equation is ambiguous [22]. The physicist's and thus our view-point is to define the solution of such "stochastic differential equations" (SDE's) to be the limit, as the the correlation time tends to zero, of the solution of a random ODE with nonwhite (colored) coefficients. In the mathematical literature on stochastic differential equations this is called the Stratonovich version of stochastic calculus [22]. When numerically solving a SDE such as (1) one cannot use standard numerical methods which assume smooth coefficients. It would also be highly impractical to use a smooth approximation of the coefficients with a small correlation time, since this requires time steps much smaller than the correlation time. Fortunately, there is an alternative formulation of the SDE, the Ito version, which overcomes this difficulty. The solutions of SDE's are Markov diffusion process which can be characterized by their diffusion and drift coefficients (also called Fokker-Planck coefficients). The so-called Ito equation 22 encodes this information in a way which leads naturally to efficient numerical schemes.

Let us briefly explain how this Ito equation is obtained. For this it is better to work with an abstract form of the starting equation (with the forcing term omitted) :

$$
\frac{d \theta}{d t}=\mathbf{M}_{\mathbf{0}} \theta+\frac{1}{\epsilon} \mathbf{M}_{\mathbf{1}}\left(\frac{t}{\epsilon^{2}}\right) \theta
$$

Here, $\mathbf{M}_{\mathbf{0}}$ is a deterministic operator and $\mathbf{M}_{\mathbf{1}}$ a random Gaussian operator with finite correlation time. The $\epsilon$-scaling is chosen in such away that, for $\epsilon \rightarrow 0$, the $\mathbf{M}_{\mathbf{1}}$ operator becomes deltacorrelated in time. Just as ordinary white noise may be written as the derivative, in the sense of distributions, of the Brownian motion process, we can write

$$
\lim _{\epsilon \rightarrow 0} \frac{1}{\epsilon} \mathbf{M}_{\mathbf{1}}\left(\frac{t}{\epsilon^{2}}\right)=\frac{\mathbf{d} \mathbf{W}}{d t},
$$

where $\mathbf{W}(\mathbf{t})$ is an operator-valued Brownian motion. The Ito SDE associated to (8) is

$$
d \theta=\left(\mathbf{M}_{\mathbf{0}}+\mathcal{D}\right) \theta+\mathbf{d} \mathbf{W} \theta
$$

where

$$
\mathcal{D}=\int_{0}^{\infty}\left\langle\mathbf{M}_{\mathbf{1}}(\mathbf{s}) \mathbf{M}_{\mathbf{1}}(\mathbf{0})\right\rangle d s
$$

is called the drift operator. The presence of the drift term in (10) is easily understood : a Neumann expansion of $\theta(t+d t)-\theta(t)$ in powers of $\mathbf{M}_{1}$, starting from (8), gives a second-order term, which is $O\left(d t^{2}\right)$ for fixed $\epsilon$ but becomes $\mathcal{D} \theta d t$ when the limit $\epsilon \rightarrow 0$ is taken. The drift term may also be obtained by using the Ito formula [22] or Gaussian integration by parts (see, e.g., Ref. [2], Section 4.1). The term $\mathbf{d} \mathbf{W} \theta$ is called the diffusion term. Since Brownian motion has independent increments over nonoverlapping intervals, the operator $\mathbf{d} \mathbf{W}$, which is $O(\sqrt{d t})$ and has zero mean, is independent of the past and, thus, of $\theta$. It follows that the diffusion term has vanishing mean. Hence, the mean of $\theta$ satisfies

$$
\frac{d\langle\theta\rangle}{d t}=\left(\mathbf{M}_{\mathbf{0}}+\mathcal{D}\right)\langle\theta\rangle
$$


The Ito SDE associated to (州) reads:

$$
\begin{aligned}
d \theta_{m}(t) & =\left(\left(a_{m} b_{m+1}+b_{m} c_{m-2}\right) D_{m-1}+\left(-a_{m} c_{m-1}+b_{m+2} c_{m}\right) D_{m+1}\right. \\
& \left.+a_{m-1} b_{m} D_{m-2}-a_{m+1} c_{m} D_{m+2}-\kappa k_{m}^{2}\right) \theta_{m}(t) d t \\
& +i\left[a_{m}\left(\theta_{m+1}^{*}(t) \alpha_{m-1} d W_{m-1}^{*}(t)-\theta_{m-1}^{*}(t) \alpha_{m+1} d W_{m+1}^{*}(t)\right)\right. \\
& +b_{m}\left(\theta_{m-1}^{*}(t) \alpha_{m-2} d W_{m-2}^{*}(t)+\theta_{m-2}^{*}(t) \alpha_{m-1} d W_{m-1}(t)\right) \\
& \left.+c_{m}\left(\theta_{m+2}^{*}(t) \alpha_{m+1} d W_{m+1}(t)+\theta_{m+1}^{*}(t) \alpha_{m+2} d W_{m+2}^{*}(t)\right)\right] \\
& +\delta_{m, 1} d W_{f}(t) .
\end{aligned}
$$

Here, $\alpha_{m}=k_{m}^{\xi / 2}, D_{m}=\alpha_{m}^{2} / 2$, the $W_{m}(t)$ 's and $W_{f}(t)$ are independent identically distributed complex-valued Brownian motion functions, normalized in such a way that $\left\langle\left|W_{m}(t)\right|^{2}\right\rangle=\left\langle\left|W_{f}(t)\right|^{2}\right\rangle=$ $t$. It is noteworthy that the drift term in (13), the top two lines on the rhs, which involves the sum of the energies in the neighboring and next-neighboring shells, may be viewed as an eddy-diffusivity term. For Kraichnan's original equation (1), the Ito equation approach has a scale-independent eddy diffusivity [25]. Here, it is proportional to $k_{n}^{-\xi}$. The difference stems from the absence, in the shell model of direct interactions between widely separated scales. We also observe that, if we had used the original model of Ref. [21] with complex conjugates on all the $u$ factors, the drift operator would involve nondiagonal elements coupling different shells, a situation we avoided.

\section{Equations for the second and fourth-order moments}

In this and the following sections we are interested in the scaling behavior of the $p$-th-order structure functions:

$$
\left\langle\left(\theta_{m} \theta_{m}^{*}\right)^{p / 2}\right\rangle \propto k_{m}^{-\zeta_{p}}
$$

where $\zeta_{p}$ is called the scaling exponent of order $p$. If $\zeta_{2 p}=p \zeta_{2}$ the structure functions are said to have a normal scaling. If $\rho_{2 p}=\zeta_{2 p}-p \zeta_{2} \neq 0$ the scaling of the structure function of order $2 p$ is said to be anomalous.

It is well known that from a linear stochastic differential equation with white-noise coefficients it is possible to obtain exact equations for moments of arbitrary order [5, 7]. For example, from the abstract equation (8) one derives the closed equation for the first-order moment (12). Higher order quantities such as $\theta \otimes \theta, \theta \otimes \theta \otimes \theta \otimes \theta, \ldots$ satisfy also linear stochastic differential equations with white-noise coefficients, from which closed equations can be obtained for $\langle\theta \otimes \theta\rangle,\langle\theta \otimes \theta \otimes \theta \otimes \theta\rangle$, etc. . Such equations may also be obtained by use of Ito calculus [22].

In the shell-model context the moment equations become excessively cumbersome beyond order four. We have obtained the closed equations for

$$
E_{m} \equiv\left\langle\theta_{m} \theta_{m}^{*}\right\rangle \quad \text { and } \quad P_{l m}=\left\langle\theta_{l} \theta_{l}^{*} \theta_{m} \theta_{m}^{*}\right\rangle .
$$

The general structure of these equations is as follows

$$
\begin{aligned}
\dot{E}_{l} & =\left(-2 \kappa k_{m}^{2} \delta_{l, m}+A_{l, m}\right) E_{m}+F_{l} . \\
\dot{P}_{l m} & =\left[-2 \kappa\left(k_{l}^{2}+k_{m}^{2}\right) \delta_{l, n} \delta_{m, j}+B_{l m, n j}\right] P_{n j}+G_{l m} .
\end{aligned}
$$


The equations are written in explicit form in the Appendix B. Here, we stress a few important properties. The forcing $F_{m}$ is restricted only to the first shell $m=1$. The matrix $A_{l, m}$ is symmetric band diagonal with bandwidth five and has the following scaling law:

$$
A_{l+s, m+s}=k_{s}^{2-\xi} A_{l, m}
$$

Similarly,

$$
B_{l+s ; m+s, n+s ; j+s}=k_{s}^{2-\xi} B_{l m, n j}
$$

Straightforward scaling arguments indicate that (16) and (17) may possess steady-state solutions with normal scaling. Such solutions have

$$
E_{m} \propto k_{m}^{2-\xi}=k_{m}^{-\zeta_{2}}
$$

and

$$
P_{m m} \propto k_{m}^{-\zeta_{4}}
$$

with $\zeta_{4}=2 \zeta_{2}$. In order to find what kind of scaling actually holds, we now resort to numerical solutions of the moment equations (16) and (17). Since these are deterministic equations they can be solved with high accuracy at relatively low cost. This is not the case of the Monte Carlo strategy of Section 5, which allows us however, to tackle structure functions of high orders.

\section{Calculation of second and fourth-order structure func- tions from the moment equations}

For the numerical solution of (16) and (17) we used a 19-shell truncation with values of $\kappa$ chosen in such a way that the diffusive cutoff is well within the available range of shells. The solutions were obtained by time-marching until the steady state is reached. Such a steady state is necessarily stable. The scaling parameter $\xi$ was varied in the range $0.2 \leq \xi \leq 2$.

We found that the second order structure function $E_{m}$ displays always normal scaling with

$$
\zeta_{2}=2-\xi
$$

the same value as obtained by Kraichnan [5]. To investigate the nature of the scaling of the fourthorder structure function $P_{m m}$, we plotted it against the second-order structure function, following the, now standard, Extended Self Similarity (ESS) procedure (see, Ref. [24]). This gave us the anomalous part $\rho_{4}=\zeta_{4}-2 \zeta_{2}$ of the scaling exponent, plotted in Fig. 1 against $\xi$. It is clearly seen that the scaling is anomalous $\left(\rho_{4}>0\right)$, the anomaly is a decreasing function of $\xi$ and disappears as $\xi \rightarrow 2$, the "laminar" limit, as in Ref. 四.

For small values of $\xi$, interactions become more and more nonlocal and a higher number of shells would be needed to have a sufficiently large inertial range. (The number of shells in the "intermediate inertial-dissipation range", in which the local Reynolds number is $O(1)$, grows like 
$1 / \xi$.) Even though our shell model has only local interactions, purely kinematic effects introduce important long-range diffusive corrections in the limit $\xi \rightarrow 0$. This is why we restricted our calculations of $\rho_{4}$ for $\xi$ ranging from 0.2 to 2.0 .

The fact that normal scaling is obtained for the second-order structure function is not very surprising, this being exactly the same situation as for Kraichnan's original problem. It is easily checked that the operator $A$ appearing in (16) has equipartition solutions $\left(E_{m}\right.$ independent of $m$ ) in its null-space, both for the full operator and its $N$-shell truncations. Such equipartition solutions have no associated passive scalar energy flux and cannot bring about anomalous scaling.

Let us now consider the anomalous scaling for the fourth-order structure function which are connected to the properties of the operator $B \equiv B_{l m, n j}$. The operator $B$ has again equipartition solutions in its null-space, which cannot cause anomalous scaling. We have checked numerically that finite-shell truncations of $B$ have no other eigenvectors in their null-space. This, only superficially contradicts the interpretation of anomalous scaling as arising from the zero modes of the inertial

operator describing the evolution of moments [6, 10]. In our case there are no anomalous zeromodes of the operator $B$ but still we see numerically a very clean intermittent behavior. Where does the observed anomaly come from? One can imagine two interpretations. The first one rests on the physical observation that the operator $B$ is naturally long-range: it mixes inertial scales with cut-offs at both the UV and IR ends. Therefore, its inverse involves a nontrivial mixture of contributions from very different scales. This mixture could be the cause of anomalous scaling, thereby defeating naive (local) dimensional analysis. The second interpretation is to imagine that quasi-zero modes (which would become true zero-modes in the limit of an infinite inertial range) are already dominating the inverse of $B$. The two interpretations are not in contradiction. Indeed, our truncated system, being always influenced by UV and IR cut-offs, it naturally takes into account boundary conditions and therefore never shows true zero-modes. A similar scenario takes place in Ref. [6], where zero modes in the infinite dimensional function space have to be matched with IR and UV physical cut-offs.

Let us finally remark, that the analysis of the relevant eigenvectors in the eigenspace of the operator $B$ is highly complicated due to the fact that we are looking at the cone of positive functions, which is not a linear space.

\section{Monte Carlo simulations for structure functions}

As we noted, the moment-equation strategy becomes inpractical for determining structure functions beyond the fourth order. We therefore resort to Monte Carlo simulations of the stochastic shell model in its Ito version (13). We used the "weak-order-one Euler" scheme, the details of which may be found in Appendix A. Roughly, this means interpreting the Ito equation (13) as a time-difference equation. This scheme is of order one (in the time step $\Delta t$ ) for averaged quantities such as the structure functions. Averages are calculated as time averages, assuming ergodicity (we checked that changes in the seed of the random generator do not affect the results). Integrating over a large number of realizations is thus equivalent to integrating over many large-eddy turnover times. 
First we validated our Monte Carlo simulation by comparison with the results for the second and fourth-order structure functions obtained from the moment equations in the previous section. The comparison can be seen in Fig. 2. The agreement of the Monte Carlo and moment based calculations is comparable to the machine precision (single precision). We mention that, in order, to maximize the extent of the inertial range and avoid an inertial-diffusive range with algebraic falloff of the passive scalar spectrum [25], we assumed an exponential cutoff on the velocity spectrum.

In all calculations 19 shells were used, the molecular diffusivity was varied between $2^{-10}$ and $2^{-8}$ and the time step between $2^{-29}$ and $2^{-25}$. In order to give an example of the quality of the scaling, we show in Fig. 3 the $\log -\log$ plot of the $4^{\text {th }}, 6^{\text {th }}$ and $8^{\text {th }}$ structure function versus the $2^{\text {nd }}$ order structure function. This is done by ESS in order to improve the scaling.

Fig. 4 shows the scaling exponents $\zeta_{p}$ determined by least square fits using ESS up to order $p=16$ for $\xi=2 / 3$. The error bars are obtained from the least square fits. In all the calculations the inertial range included eight shells or more. When halving the number of samples in our statistics, we found that the values obtained remained well within the error bars. The graph of $\zeta_{p}$ appears to be linear at values of $p$ beyond 8 , with a slope of about 0.29 . We do not rule out that the asymptotic linear trend is an artifact due to insufficient statistics. We observe that the $\zeta_{p}$ 's obtained with our Gaussian white-in-time velocity are less anomalous than those reported in Ref. [21], where the velocity was already multifractal.

\section{Conclusions}

We proposed and studied a shell model for a randomly advected passive scalar. We suggest that anomalous scaling of structure functions of order greater than two is connected to nontrivial dependence of inertial-range properties on both integral and diffusive scales. This is shown, for example, in the closed equation for the fourth-order moments $\left\langle\theta_{m} \theta_{m}^{*} \theta_{l} \theta_{l}^{*}\right\rangle$, where inertial-range shell correlations depend explicitly on correlations between distant shells with $m \ll l$. The main advantage of our model is that reliable numerical simulations become feasible. We numerically estimated anomalous exponents for structure functions up to order 16 and for various scaling exponents $\xi$ of the velocity field $(0.2 \leq \xi \leq 2)$. Comparing our numerical results with those obtained in a similar passive shell model [21], but advected by a multifractal velocity field, we find large quantitative differences. This is due to the obvious fact that the $\theta$-statistics are strongly correlated to the statistics of the advecting field. Nevertheless, the possibility of writing down closed equations for correlations of any order could help in the understanding of intermittency in more general cases. Unfortunately, we do not see how to implement in our model the kind of perturbation expansion done in Refs. [6, 10]. The main difficulty is the lack of long-range interactions in our model which forbids a proper definition of an eddy diffusivity in the limit $\xi \rightarrow 0$. Some long-range shell models should be introduced and studied if one wants to follow this path.

Another interesting question is to find the simplest passive-scalar shell model which has inertialrange intermittency. For example, it is easy to extract from (44) an even simpler shell model, by retaining only terms with $\theta_{m-2}^{*}(t) u_{m-1}(t)$ and $\theta_{m+2}^{*}(t) u_{m+1}(t)$ in the advecting part. This model has 
the same properties as listed in the Introduction and some preliminary numerical results indicate that it has similar intermittency corrections.

Let us, finally, remark that, up to now, all the existing work on anomalies driven by stochastic velocity field was rooted in some kind of analysis performed in the physical space. In contrast, our shell model exists only in a kind of Fourier space. Understanding the anomalous scaling in our shell model at a phenomenological level could help in devising a phenomenology of intermittency. For example, ideas connected to the popular inertial-range cascade picture could be usefully revisited.

Acknowledgements We would like to express our gratitude to M. Vergassola for many stimulating and interesting discussions. We have also benefited from extensive discussions with R. Benzi, U. Frisch and A. Noullez. This work was supported by the French Ministère de la Recherche et de la Technologie, by the European Union (Human Capital and Mobility ERBCHRXCT920001) and by the GDR Mécanique des Fluides Numérique. L.B. would like to acknowledge partial support by Ministère de l'Enseignement Superieur et de la Recherche (France) and the Observatoire de la Côte d'Azur where this work was completed. 


\section{Appendices}

\section{A The Euler scheme}

The weak-order-one Euler scheme [22] associated to the passive scalar shell model in its Ito form (4) reads :

$$
\begin{aligned}
\theta_{m}^{n+1} & =\left\{1+\left(\left(a_{m} b_{m+1}+b_{m} c_{m-2}\right) D_{m-1} \Delta t+\left(-a_{m} c_{m-1}+b_{m+2} c_{m}\right) D_{m+1} \Delta t\right.\right. \\
& \left.\left.+a_{m-1} b_{m} D_{m-2} \Delta t-a_{m+1} c_{m} D_{m+2} \Delta t-\kappa k_{m}^{2} \Delta t\right)\right\} \theta_{m}^{n} \\
& +i\left[a_{m}\left(\theta_{m+1}^{n *} \alpha_{m-1} \Delta W_{m-1}^{n *}-\theta_{m-1}^{n *} \alpha_{m+1} \Delta W_{m+1}^{n *}\right)\right. \\
& +b_{m}\left(\theta_{m-1}^{n *} \alpha_{m-2} \Delta W_{m-2}^{n *}+\theta_{m-2}^{n *} \alpha_{m-1} \Delta W_{m-1}^{n}\right) \\
& \left.+c_{m}\left(\theta_{m+2}^{n *} \alpha_{m+1} \Delta W_{m+1}^{n}+\theta_{m+1}^{n *} \alpha_{m+2} \Delta W_{m+2}^{n *}\right)\right] \\
& +f \delta_{m, 1} \Delta W_{f}^{n} .
\end{aligned}
$$

Here,

$$
\Delta W_{m}^{n}=\sqrt{\Delta t} \eta_{m}^{n}
$$

where the $\eta_{m}^{n}$ are independent identically distributed complex random variables of the form $a+i b$ where $a$ and $b$ are independent Bernoulli variables with values $\pm 1 / \sqrt{2}$. The Bernoulli variables are numerically generated by a linear feed-back shift register random number generator (see, e.g., [26]).

The choice of Bernoulli variables rather than Gausssian variables is particularly convenient for numerical purposes. It ensures that averaged quantities such as moments are correct to first order in $\Delta t$, a choice consistent with the scheme.

\section{B Moment equations}

We give hereafter the detailed form of the equation for the second order moments $E_{m} \equiv\left\langle\theta_{m} \theta_{m}^{*}\right\rangle$ and the fourth-order moment $P_{l m} \equiv\left\langle\theta_{l} \theta_{l}^{*} \theta_{m} \theta_{m}^{*}\right\rangle$.

$$
\begin{aligned}
\dot{E}_{m} & =+\left(-2 \kappa k_{m}^{2}+A_{m, m}\right) E_{m}+A_{m, m-2} E_{m-2}+A_{m, m-1} E_{m-1} \\
& +A_{m, m+1} E_{m+1}+A_{m, m+2} E_{m+2}+F_{m} . \\
\dot{P}_{l, m} & =\left[1-\left(2 \kappa\left(k_{m}^{2}+k_{l}^{2}\right)-A_{m, m}-A_{l, l}+\delta_{l-1, m} 2 A_{m+1, m}+\delta_{l-2, m} 2 A_{m+2, m}\right)\right] P_{m, l} \\
& +A_{m, m-2} P_{l, m-2}+A_{m, m-1} P_{l, m-1}+A_{m, m+1} P_{l, m+1}+A_{m, m+2} P_{l, m+2} \\
& +A_{l, l-2} P_{m, l-2}+A_{l, l-1} P_{m, l-1}+A_{l, l+1} P_{m, l+1}+A_{l, l+2} P_{m, l+2}^{n} \\
& +\delta_{l, m} 2\left(A_{m, m-2} P_{m, m-2}+A_{m, m-1} P_{m, m-1}+A_{m, m+1} P_{m, m+1}+A_{m, m+2} P_{m, m+2}\right) \\
& +\left(\delta_{1, m} E_{m}+\delta_{1, l} E_{l}+\delta_{1, m} \delta_{1, l} E_{1}\right) F_{1} .
\end{aligned}
$$


The following notation has been used.

$$
\begin{aligned}
F_{1} & =\frac{1}{2} \\
F_{l} & =0 \quad \forall \quad l \neq 1 \\
A_{m, m-2} & =2 b_{m}^{2} D_{m-1} \\
A_{m, m-1} & =2\left(a_{m}^{2} D_{m+1}+b_{m}^{2} D_{m-2}\right) \\
A_{m, m+1} & =2\left(a_{m}^{2} D_{m-1}+c_{m}^{2} D_{m+2}\right) \\
A_{m, m+2} & =2 c_{m}^{2} D_{m+1}, \\
A_{m, m} & =-A_{m+2, m}-A_{m+1, m}-A_{m-1, m}-A_{m-2, m} .
\end{aligned}
$$




\section{References}

[1] K.R. Sreenivasan, Proc. Roy. Soc. Lond. A434, 165 (1991).

[2] U. Frisch, Turbulence Cambridge University Press (1995).

[3] A.M. Obukhov, Izv. Akad. SSSR, Serv. Geogr. Geofiz. 13, 58 (1949); S. Corrsin, J. Appl. Phys. 22, 469 (1951).

[4] R.H. Kraichnan, Phys. Rev. Lett. 72, 1016 (1994).

[5] R.H. Kraichnan, Phys. of Fluids 11, 945 (1968).

[6] K. Gawedzki and A. Kupiainen, Phys. Rev. Lett. 75, 3834 (1995).

[7] R.H. Kraichnan, V. Yakhot and S. Chen, Phys. Rev. Lett. 75, 240 (1995).

[8] M Chertkov, G. Falkovich, I. Kolokolov and V. Lebedev, Phys. Rev. E52, 4924 (1995); M. Chertkov and G. Falkovich, "Anomalous scaling exponents for a white-advected passive scalar", submitted to Phys. Rev. Lett. (1996).

[9] M. Chertkov, G. Falkovich and V. Lebedev, "Non-universality of the scaling exponents of a passive scalar advected by a random flow", submitted to Phys. Rev. Lett. (1996).

[10] D. Bernard, K. Gawedzki and A. Kupiainen, "Anomalous scaling in the N-point functions of passive scalar", preprint (1996) chao-dyn 9601018.

[11] A.L. Fairhall, O. Gat, V. L'vov and I. Procaccia, "Anomalous scaling in a model of passive scalar advection: exact results" Phys. Rev. E in press (1996).

[12] G. L. Eyink, "Intermittency and anomalous scaling of passive scalars in any space dimension", preprint (1996).

[13] M. Vergassola, "Anomalous scaling for passively advected magnetic field", Phys. Rev. E in press (1996).

[14] L. Kadanoff, Physics Today 48, 11 (1995).

[15] E.B. Gledzer, Sov. Phys. Dokl. 18, 216 (1973).

[16] M. Yamada and K. Ohkitani, J. Phys. Soc. Jpn. 56, 4210 (1987); Prog. Theor. Phys. 79, 1265 (1988).

[17] M.H. Jensen, G. Paladin and A. Vulpiani, Phys. Rev. A43, 798 (1991); D. Pisarenko, L. Biferale, D. Courvoisier, U. Frisch and M. Vergassola, Phys. Fluids A5, 2533 (1993).

[18] R. Benzi, L. Biferale and G. Parisi, Physica D65, 163 (1993).

[19] L. Kadanoff, D. Lohse, J. Wang and R. Benzi, Phys. Fluids 7, 617 (1995). 
[20] R. Benzi, L. Biferale, R. Kerr and E. Trovatore, Phys. Rev. E in press (1996).

[21] M.H. Jensen, G. Paladin and A. Vulpiani, Phys. Rev. A45, 7214 (1992).

[22] P. E. Kloeden and E. Platen, Numerical Solution of Stochastic Differential Equations, Springer Verlag (1992).

[23] J. G. Gaines and T. J. Lyons, SIAM J. Appl. Math 54, 1132 (1994).

[24] R. Benzi, S. Ciliberto, R. Tripiccione, C. Baudet, F. Massaioli and S. Succi, Phys. Rev. E48, R29 (1993).

[25] U. Frisch and A. Wirth, "Batchelor inertial-diffusive range for a passive scalar advected by a white-in-time velocity field: spectrum and pdf", preprint (1996).

[26] D. E. Knuth, Seminumerical Algorithms, Addison Wesly (1981) 